\title{
Stellar Population Models with Variable Element Abundance Ratios
}

\author{
Daniel Thomas, Claudia Maraston, Ralf Bender \\ MPE, Giessenbachstraße, 85748 Garching, Germany \\ Universitäts-Sternwarte, Scheinerstraße 1, 81679 München, Germany
}

\begin{abstract}
We present a comprehensive set of new generation stellar population models of Lick absorption line indices, which for the first time include element abundance ratios different from solar. We computed the 21 Lick indices $\mathrm{CN}_{1}, \mathrm{CN}_{2}, \mathrm{Ca} 4227, \mathrm{G} 4300, \mathrm{Fe} 4383, \mathrm{Ca} 4455, \mathrm{Fe} 4531, \mathrm{C}_{2} 4668, \mathrm{H} \beta, \mathrm{Fe} 5015, \mathrm{Mg}_{1}$, $\mathrm{Mg}_{2}, \mathrm{Mg}_{b}, \mathrm{Fe} 5270, \mathrm{Fe} 5335, \mathrm{Fe} 5406, \mathrm{Fe} 5709, \mathrm{Fe} 5782, \mathrm{Na} \mathrm{D}, \mathrm{TiO}_{1}$, and $\mathrm{TiO}_{2}$, in the wavelength range $4000 \lesssim \lambda \lesssim 6500 \AA$. Models are provided with: $[\alpha / F e]=$ $0.0,0.3,0.5,[\alpha / C a]=-0.1,0.0,0.2,0.5$, and $[\alpha / N]=-0.5,0.0 ;$ ages from 1 to $15 \mathrm{Gyr}$; total metallicities from $1 / 200$ to 3.5 solar $(-2.25 \leq[Z / H] \leq 0.67)$.
\end{abstract}

The models are based on the evolutionary synthesis technique described in Maraston (1998). The $\alpha / F e$ enhanced mixtures are obtained by increasing the abundances of $\alpha$-group elements and by decreasing the abundances of the Fe-peak elements, such that total metallicity is conserved. The impact from these element abundance variations on the absorption line indices is taken from Tripicco \& Bell (1995), using an extension of the method introduced by Trager et al. (2000). Most importantly, we take into account that the empirical stellar libraries used to compute model indices follow the chemical enrichment history of the Milky Way, and are therefore biased towards super-solar $\alpha / \mathrm{Fe}$ ratios at sub-solar metallicities. We corrected for this bias, so that the models presented here have well-defined $\alpha / F e$ ratios at all metallicities.

We take particular care at calibrating the models with galactic globular clusters, for which ages, metallicities, and element abundance ratios are known from independent sources. Our $\alpha / F e$ enhanced models with $[\alpha / F e]=0.3$ (and 12 Gyr age) perfectly reproduce the positions of the globular cluster data in the $\mathrm{Mg}_{1}-\langle\mathrm{Fe}\rangle$ diagram up to solar metallicities (see also Maraston et al. 2003). The total metallicities for the sample clusters that we derive from these indices are in excellent agreement with the Zinn \& West (1984) metallicity scale. We point out that the latter most likely reflects total metallicity rather than iron abundance, because it is obtained essentially by averaging the abundances derived from the $\mathrm{Mg}$ triplet near $5175 \AA$ and the Fe blend at $5270 \AA$. This aspect needs to be emphasized, as with the $\alpha / F e$ enhanced models we are now in the position to distinguish total metallicity $[\mathrm{Z} / \mathrm{H}]$ and iron abundance $[\mathrm{Fe} / \mathrm{H}]$.

By means of our calibrated $\alpha / F e$ enhanced models, we confirm that the index $[\mathrm{MgFe}]$, suggested by González (1993) to balance $\alpha / \mathrm{Fe}$ ratio effects, is almost independent of $\alpha / \mathrm{Fe}$. As it modestly decreases with increasing $\alpha / F e$, however, we define the slightly modified index

$[\mathrm{MgFe}]^{\prime} \equiv \sqrt{M g_{b}(0.72 \cdot \mathrm{Fe} 5270+0.28 \cdot \mathrm{Fe} 5335)}$ which is completely independent of $\alpha / F e$, and hence an even better tracer of total metallicity. We further show that the linear correlation between $\mathrm{Mg}_{2}$ and metallicity at old ages derived 
empirically by Brodie \& Huchra (1990) is valid up to $\sim 1 / 3$ solar metallicity, but underpredicts $\mathrm{Mg}_{2}$ indices at metallicities above that threshold.

It turns out to be hard to find indices that correlate with $\alpha / F e$ as well as the intensively studied indices $\mathrm{Mg}_{1}, \mathrm{Mg}_{2}$, and $\mathrm{Mg}_{b}$. Promising alternatives are the blue indices $\mathrm{CN}_{1}$ and $\mathrm{CN}_{2}$ that also increase with increasing $\alpha / F e$ ratio, mainly because of an anti-correlation with $\mathrm{Fe}$ abundance. With the caveat that $\mathrm{CN}_{1}$ and $\mathrm{CN}_{2}$ are additionally sensitive to $\mathrm{C}$ and Nabundances, they can be regarded to be complementary to the indices $\mathrm{Mg}_{1}, \mathrm{Mg}_{2}$, and $\mathrm{Mg}_{b}$. Alternatives to the iron indices Fe5270 and Fe5335, the strengths of which decrease with increasing $\alpha / F e$ ratio, are easier to find. The best cases are the indices Fe 4383 , Fe4531, Fe5015, and Fe5709.

The indices $\mathrm{CN}_{1}, \mathrm{CN}_{2}$, and $\mathrm{Ca} 4227$ of globular clusters are very interesting, particular cases. We find that the relatively strong $\mathrm{CN}$ features observed in globular clusters require models in which nitrogen is enhanced by a factor three relative to the $\alpha$-elements, hence $[\alpha / N]=-0.5$. This is in agreement with early suggestions by D'Antona (2003), that stars in globular clusters are nitrogen enriched by a previous generation of stars. The good calibration of other indices like $\mathrm{Mg}_{1}, \mathrm{Mg}_{b}$ or $\langle\mathrm{Fe}\rangle$ is not affected by a variation of the $[\alpha / N]$ ratio, as these indices are not sensitive to nitrogen abundance. We note that an enhancement of carbon abundance, instead, would lead to serious inconsistencies with $\mathrm{Mg}_{1}$. Interestingly, also Ca4227 is sensitive to nitrogen abundance, and the globular cluster data of this index are also best reproduced by the model with increased nitrogen abundance.

To conclude, the stellar population models presented here make it possible, for the first time, to study in detail individual element abundance ratios of unresolved stellar populations (Thomas et al. 2003b). In particular, total metallicity is now a well-defined quantity.

The models are published in Thomas et al. (2003a). Tables are available electronically via anonymous ftp at ftp.mpe.mpg.de in the directory people/dthomas/SSPs. They are also available via WWW by going to ftp://ftp.mpe.mpg.de/people/dthomas/SSPs.

\section{References}

Brodie J., Huchra J., 1990, ApJ, 362, 503

Da Costa, G. S., D'Antona, F. and Gratton, R. G. 2003, This volume, JD 4

González, J. J. 1993, PhD thesis, University of Santa Cruz

Maraston C. 1998, MNRAS, 300, 872

Maraston C., Greggio L., Renzini A. et al. 2002, A\&A, 400, 823

Trager S. C., Faber S. M., Worthey G., González J. J. 2000, AJ, 119, 164

Tripicco M. J., Bell R. A. 1995, AJ, 110, 3035

Thomas, D., Maraston, C., Bender, R. 2003a, MNRAS, 339, 897

Thomas, D., Maraston, C., Bender, R. 2003b, MNRAS, 343, 279

Zinn R., West M. J. 1984, ApJS, 55, 45 